\title{
EVALUACIÓN FORMATIVA Y CONSERVATORIOS SUPERIORES: OBLIGADOS A ENTENDERSE. (PROPUESTA DE INTERVENCIÓN)
}

Formative evaluation and higher conservatories: forced to get along well. (Proposal of intervention)

Avaliação formativa e conservatórios superiores: forçados a entender-se. (Proposta de intervenção)

Pablo Fernández Baladrón (1)

Inés María Monreal Guerrero (2)

(1) Escuela de Música Creativa (Madrid). Teléfono: +34 618257495. Correo: p.fernandez.baladron@gmail.com

(2) Universidad de Valladolid. Teléfono: +34 615208622. Correo: ines.monreal@mpc.uva.es

\section{Resumen}

Los conservatorios superiores de música precisan de sistemas de evaluación adaptados al constructivismo social, que evalúen los procesos de aprendizaje autónomo del estudiante y su rendimiento a lo largo del curso escolar, alejándose de procesos pedagógicos basados en el sistema de la clase magistral unidireccional y conductista como única vía de acceso al conocimiento. Las instituciones educativas españolas deben garantizar que dichos centros sigan una línea de trabajo constructivista y dialógica que garantice los principios que la sociedad de hoy exige. El objetivo de este trabajo es el diseño de una propuesta de evaluaciónn formativa para un Conservatorio de Música ajustada a los cambios que se implementan utilizando distintas metodologías activas de aprendizaje. Estos cambios podrían resumirse en: atención y tutorización individualizada centrando la docencia en los alumnos, el profesor como guía del proceso de aprendizaje, relación dialéctica bidireccional entre profesor y alumno, y evaluación formativa y participativa.

Palabras clave: evaluación compartida; evaluación formativa; conservatorios; educación musical.

\begin{abstract}
The higher music conservatories need evaluation systems adapted to social constructivism, which evaluate the autonomous learning processes of the student and their performance throughout the school year, moving away from pedagogical processes Evaluación formativa y conservatorios superiores: obligados a entenderse. (Propuesta de intervención)


based on the unidirectional master class system such as the only way of access to knowledge. The spanish educational institutions must guarantee that these centres and their different methodologies follow a constructivist and dialogical line of work that guarantees the principles that today's society demands. The objective of this work is to design a formative evaluation proposal for a Conservatory of Music adjusted to the changes that are implemented using different active learning methodologies. These changes could be summarized: individual attention and tutoring focusing on teaching on students, the teacher as a guide to the learning process, a two-way dialectic relationship between teacher and student, and formative and participatory evaluation.

Keywords: shared evaluation; formative evaluation; conservatories; music education.

\section{Resumo}

Os conservatórios superiores de música precisam de sistemas de avaliação adaptados ao construtivismo social, que avaliem os processos de aprendizagem autónoma dos alunos e seu desempenho ao longo do ano escolar, afastando-se de processos pedagógicos baseados no sistema de aula magistral unidirecional e diretiva como única via de acesso ao conhecimento. As instituições educativas espanholas devem garantir que estes centros sigam uma linha de trabalho construtivista e dialógica que garanta os princípios que a sociedade atual exige. O objetivo deste trabalho é o desenho de uma proposta de avaliação formativa para um Conservatório de Música ajustado às mudanças que são implementadas face ao uso de diferentes metodologias de aprendizagem ativa. Estas mudanças podiam resumir-se em: atenção e tutoria individualizada, centrando o ensino nos estudantes, o professor como guia do processo de aprendizagem, relação dialética bidirecional entre professor e aluno, e avaliação formativa e participativa.

Palavras-chave: avaliação partilhada; avaliação formativa; conservatórios; educação musical.

\section{Introducción}

Tradicionalmente, los sistemas de evaluación en los conservatorios de música han estado centrados en la calificación de las interpretaciones como principal prueba donde constatar el aprendizaje, y en la clase magistral como medio de adquisición del conocimiento.

Evaluación formativa y conservatorios superiores: obligados a entenderse. (Propuesta de intervención) 
Con respecto a la legislación educativa española en el ámbito de las enseñanzas artísticas, se aprecia que dichas enseñanzas no están circunscritas a la LOMCE (2013), sino a la LOE (2006). La legislación obliga a los conservatorios a presentar al iniciarse el curso escolar los contenidos y procesos que serán evaluados, pero no se hace referencia a qué tipo de evaluación debe utilizarse. Es importante tener estos datos presentes de cara a la comprensión de la siguiente propuesta de intervención.

Tras la revisión bibliográfica, se ha comprobado la casi ausencia de publicaciones representativas sobre la evaluación formativa y compartida en Conservatorios Superiores de Música. A pesar de la existencia de bibliografía especializada en Evaluación Formativa y Compartida en enseñanzas universitarias $y$, en menor medida, en enseñanzas no universitarias, en concreto, se observa la falta de referencias directas sobre experiencias evaluativas formativas en Conservatorios de Música.

La Red de Evaluación Formativa y Compartida en Educación nos ha nutrido de publicaciones indexadas relacionadas con nuestro objeto de estudio. Autores como López (2006, 2009, 2010), Hamodi y López (2012) y Hamodi (2014), entre otros, muestran experiencias de aprendizaje referidos a la aplicación de procesos de evaluación formativos, relacionando participación del alumnado y rendimiento escolar con su motivación hacia el aprendizaje.

López (2006) reconoce que "la evaluación es un proceso de elaboración de un juicio de valor sobre un proceso (y/o producto de una actividad, una ejecución, un comportamiento, un trabajo) en orden a tomar una decisión sobre el mismo" (p.24).

Para ello, se expone a continuación una propuesta de intervención para adaptar la evaluación en los conservatorios de música a los sistemas de evaluación formativa y compartida.

\section{Contextualización}

La presente propuesta de intervención ha sido diseñada en el ámbito del primer curso de Grado Superior de Música en la especialidad Saxofón del Conservatorio Superior de las Islas Baleares, ubicado en Palma de Mallorca (España).

\section{Diseño y desarrollo}

En este punto, partimos del diseño de la propuesta de intervención como objetivo del trabajo. Se propone un sistema de evaluación formativa basado en el desarrollo integral

Evaluación formativa y conservatorios superiores: obligados a entenderse. (Propuesta de intervención) 
y en la autonomía del alumnado como principios, la Tabla 1 expone los instrumentos de evaluación.

Tabla 1.

Instrumentos de evaluación para el Conservatorio Superior de Música de las Islas Baleares (España).

\begin{tabular}{lll}
\hline Orales & Escritos & Observaciones \\
\hline Debates como Tertulia musical & Portafolio & Fichas de coevaluación \\
dialógica & & Fichas de autoevaluación \\
\hline
\end{tabular}

En primer lugar, el profesor debe dar cabida al debate como parte fundamental de la tertulia musical diálogica, entendida como un espacio donde alumnos, oyentes y el propio profesor compartan puntos de vista sobre composiciones musicales. Sus intervenciones y participaciones crean una atmósfera de aprendizaje dialógica como guía para crecer como artistas, donde hablar sobre aspectos técnicos, apreciaciones estilísticas y dudas y sugerencias sobre la interpretación.

La aplicación de la propuesta de intervención también incluye la integración del portafolio como instrumento de selección de evidencias y reflexiones sobre el proceso enseñanza-aprendizaje. De acuerdo con Colén, Giné e Imbernón (2006), está orientado a alcanzar la autonomía en el proceso de aprendizaje. Cada alumno puede comprobar la calidad de su aprendizaje mientras realiza esa selección que debe incluir documentos escritos, apuntes, grabaciones de actuaciones, análisis de las mismas, comentarios de sus compañeros, y aspectos a mejorar para futuras interpretaciones.

Por último, se plantea integrar las fichas de coevaluación (Ibarra, Rodríguez y Gómez, 2012) (o evaluación entre iguales) y de autoevaluación tras las interpretaciones públicas con unos ítems específicos que arrojen información valiosa para la evaluación, de forma que cada estudiante pueda comparar sus sensaciones con las impresiones del público, a menudo compuesto por perfiles diversos (como familias, profesores, compañeros, amigos).

Los sistemas de evaluación propuestos son formativos ya que, tomando como referencia a López, Monjas, Manrique, Barba y González (2008), y más tarde a López (2009) cumplen los siguientes requisitos:
A) Adecuación.
B) Relevancia.
C) Viabilidad.

Evaluación formativa y conservatorios superiores: obligados a entenderse. (Propuesta de intervención) 
D) Veracidad.

E) Formación.

F) Integración.

G) Ética.

H) Participación y negociación.

I) Autorregulación (p.97)

"Oportunidad de aprendizaje para todos los implicados", así es como Bretones (2006) concluye su definición de la evaluación compartida y que sirve de síntesis para definir la evaluación participativa propuesta, donde los alumnos desarrollan su pensamiento crítico en la auto-reflexión sobre la calidad de su aprendizaje y lo evalúan, así como su autonomía e iniciativa personal en el proceso.

Con esta propuesta, pretendemos contribuir a implementar en los Conservatorios Superiores el aprendizaje constructivista, utilizando estrategias propias de las metodologías activas y participativas donde ofrecer amplios espacios para el aprendizaje que incluyen espacios dialécticos. Cada alumno debe encontrar en los conservatorios lugares donde sentirse estimulado hacia el aprendizaje musical dialógico, donde vivir e interiorizar cada concierto y donde preguntarse qué y cómo aprende, y qué calidad tiene su proceso de aprendizaje y su interpretación en el recital.

\section{Evaluación}

Dado que aún no se ha realizado la experiencia, no hay datos sobre la evaluación de la misma.

\section{Conclusiones}

De acuerdo con el objetivo inicial de este trabajo, hemos advertido la necesidad de implementar cambios metodológicos en los sistemas de evaluación dentro de los Conservatorios Superiores de Música. Consideramos que en enseñanzas artísticas es prioritario para pasar de la cultura del examen a la cultura de la evaluación, más propia de sistemas educativos basados en el constructivismo. Esa cultura de la evaluación debería estar circunscrita a la utilización de la evaluación formativa. Con la futura puesta en práctica de esta propuesta basada en el debate como tertulia musical dialógica, en el portafolio, en las fichas de coevaluación y en las fichas de autoevaluación, los estudiantes serán conscientes de su aprendizaje y de la evaluación del mismo. Las acciones docentes propuestas se han diseñado dirigiéndose hacia un modelo de evaluación formativo y Evaluación formativa y conservatorios superiores: obligados a entenderse. (Propuesta de intervención) 
compartido que favorezcan el pensamiento crítico y la implicación del alumnado durante sus estudios de música en el Conservatorio.

\section{Referencias}

Bretones, A. (2006). La participación del alumnado en la evaluación de sus aprendizajes. Kikiriki, 65, 6-15.

Colén, M. T.; Giné, N. E, \& Imbernón, F. (2006). La carpeta de aprendizaje del alumnado universitario. Barcelona: Octaedro/ ICE UB.

Hamodi, C. \& López, A.T. (2012). La evaluación formativa y compartida en la Formación Inicial del Profesorado desde la perspectiva del alumnado y de los egresados. Psychology, Society, \& Education, 4, 1, 99-112.

Hamodi, C. (2014). La evaluación formativa y compartida en educación superior: un estudio de casos. Tesis doctoral inédita. Universidad de Valladolid.

Hamodi, C.; López, V. M.; \& López, A. T. (2014). Red de Evaluación Formativa y Compartida en Docencia Universitaria: Creación, Consolidación y Líneas de Trabajo. Revista de evaluación Educativa. Revalue, 3,1.

Ibarra-Sáiz, M., Rodríguez-Gómez, G., \& Gómez-Ruiz, M. (2012). La evaluación entre iguales: beneficios y estrategias para su práctica en la universidad. Revista de Educación, 359, 206-231.

López Pastor, V. M. (coord.). (2006). La Evaluación en Educación Física. Revisión de los modelos tradicionales y planteamiento de una alternativa: la evaluación formativa y compartida. Buenos aires: Miñó y Dávila.

López, V. M.; Monjas, R.; Manrique, J. C.; Barba, J.; \& González, M. (2008). Implicaciones de la evaluación en los enfoques de Educación Física cooperativa. El papel de la evaluación formativa y compartida en la necesaria búsqueda de coherencia. Cultura y Educación. 20, 4, 457-477.

López Pastor, V. M. (coord.). (2009). La Evaluación Formativa y Compartida en Educación Superior: propuestas, técnicas, instrumentos y experiencias. Madrid: Narcea.

López Pastor, V. M. (2010) El papel de la Evaluación Formativa en la evaluación por competencias: aportaciones de la Red de Evaluación Formativa y Compartida en Docencia Universitaria. REDU. Revista de Docencia Universitaria, 9(1), 159173.

Evaluación formativa y conservatorios superiores: obligados a entenderse. (Propuesta de intervención) 